\title{
Relative bulk and interface contributions to optical second-harmonic generation in silicon
}

\author{
H. J. Peng, * E. J. Adles, J.-F. T. Wang, and D. E. Aspnes \\ Department of Physics, North Carolina State University, Raleigh, North Carolina 27695-8202, USA
}

(Received 16 August 2005; published 14 November 2005)

\begin{abstract}
Using the simplified bond-hyperpolarizability model, we obtain analytic expressions for the first-forbidden (spatial dispersion, magnetic dipole/electric quadrupole) bulk contributions to second-harmonic generation for centrosymmetric materials. Applying these to oxidized $\mathrm{Si}$, we show theoretically and by comparison to experiment that the relative bulk contribution near $800 \mathrm{~nm}$ is minor, less than half that of the interface, but that the coherent superposition of bulk and interface contributions is important and cannot be neglected.
\end{abstract}

DOI: 10.1103/PhysRevB.72.205203

PACS number(s): 78.20.-e, 42.65.-k, 78.68.+m

Second-harmonic generation (SHG) has been widely used to study surfaces and interfaces of centrosymmetric semiconductors. ${ }^{1-4}$ For materials with inversion symmetry SHG is electric-dipole forbidden in the bulk, which makes it particularly attractive for this purpose. To facilitate interpretation of SHG data we recently developed a simplified bondhyperpolarizability model (SBHM) that incorporates the essential physics of SHG, third-(THG-), and fourth-(FHG-) harmonic generation on an atomic scale. ${ }^{5-8}$ With very few parameters the model accurately describes observed intensity anisotropies of SHG, THG, and FHG in terms of radiation from charges driven by the pump field, with motion constrained to be along the bond directions (for symmetry reasons THG requires motion in the transverse direction as well). Although highly simplified, the model embodies the bond-geometric contributions to NLO signals, and hence provides a means of assessing whether contributions other than these may be important.

For SHG some uncertainty remains. In previous work ${ }^{5,6}$ we considered only the interface contribution and obtained excellent agreement between theory and experiment. However, Lüpke et al. ${ }^{9}$ indicated that the bulk contributions could not be neglected relative to that of the interface, and in fact accounted for fully half of the observed SHG signal from oxidized Si interfaces. Here, we use the SBHM to estimate the first-forbidden bulk contributions to SHG by obtaining and evaluating analytic expressions, then directly comparing predicted anisotropies to experiment. We find that bulk contributions are indeed minor, certainly less than half the interface contributions. However, the coherent superposition of bulk and interface contributions is important and cannot be neglected.

To illustrate the origins of the various contributions and to evaluate the first-forbidden bulk contributions to SHG in centrosymmetric materials in a systematic manner, we begin with the Green-function expression for the 4-potential of the radiation from a point charge $q$ propagating in free space:

$$
\begin{aligned}
{[\phi(\vec{r}, t), \vec{A}(\vec{r}, t)]=} & \frac{1}{c} \int d^{3} r^{\prime} d t^{\prime}\left[c \rho\left(\vec{r}^{\prime}, t^{\prime}\right), \vec{J}\left(\vec{r}^{\prime}, t^{\prime}\right)\right] \\
& \times \frac{\delta\left(t-t^{\prime}-\frac{1}{c}\left|\vec{r}-\vec{r}^{\prime}\right|\right)}{\left|\vec{r}-\vec{r}^{\prime}\right|},
\end{aligned}
$$

where

$$
\begin{gathered}
\rho\left(\vec{r}^{\prime}, t^{\prime}\right)=q \delta\left[\vec{r}^{\prime}-\vec{r}_{o}-\Delta \vec{r}\left(t^{\prime}\right)\right] ; \\
\vec{J}\left(\vec{r}^{\prime}, t^{\prime}\right)=\rho\left(\vec{r}^{\prime}, t^{\prime}\right) \frac{d}{d t^{\prime}} \Delta \vec{r}\left(t^{\prime}\right) ;
\end{gathered}
$$

where $\vec{r}_{o}$ is the equilibrium position of $q$ and $\Delta \vec{r}$ is a small displacement. We assume that $q$ is located in the bond $j$ with the direction of the bond specified by the unit vector $\hat{b}_{j}$. For interface contributions we have shown that good results are obtained by assuming that the interface is atomically smooth and the directions of the bonds there are the same as those in the bulk. ${ }^{5-8}$ Interface roughness is known to be a factor for FHG, but for SHG we would expect it mainly to affect the scaling factors and not the azimuthal dependence of the SHG intensity. Since the data do not contain enough information to allow us to evaluate the scaling factors, we do not pursue the point further. For the bulk contributions the bond directions are known, so this does not need to be considered an assumption.

For SHG $\Delta \vec{r}_{j}\left(t^{\prime}\right)$ has the form

$$
\Delta \vec{r}_{j}\left(t^{\prime}\right)=\left(\Delta r_{j 1} e^{-i \omega t^{\prime}}+\Delta r_{j 2} e^{-i 2 \omega t^{\prime}}\right) \hat{b}_{j}
$$

In the SBHM the time-independent quantities $\Delta r_{j 1}$ and $\Delta r_{j 2}$ are solutions of the one-dimensional force equation

$$
\begin{gathered}
F=m \frac{d^{2}}{d t^{\prime 2}}\left(\Delta r_{j 1} e^{-i \omega t^{\prime}}+\Delta r_{j 2} e^{-i 2 \omega t^{\prime}}\right) \\
=-m \omega^{2} \Delta r_{j 1} e^{-i \omega t^{\prime}}-4 m \omega^{2} \Delta r_{j 2} e^{-i 2 \omega t^{\prime}} \\
=q\left(\hat{b}_{j} \cdot \vec{E}\right) e^{i \vec{k}_{o} \cdot \vec{r}_{o}-i \omega t^{\prime}}\left[1+i\left(\vec{k}_{o} \cdot \hat{b}\right) \Delta r_{j 1} e^{-i \omega t^{\prime}}\right] \\
-\kappa_{1}\left(\Delta r_{j 1} e^{-i \omega t^{\prime}}+\Delta r_{j 2} e^{-i 2 \omega t^{\prime}}\right)-\kappa_{2} \Delta r_{j 1}^{2} e^{-i 2 \omega t^{\prime}} .
\end{gathered}
$$

The gradient projection $\left(\vec{k}_{o} \cdot \hat{b}\right)$ of the driving field gives rise to the contribution from spatial dispersion. The quantities $\kappa_{1}$ and $\kappa_{2}$ describe the linear (Hooke's law) and second-order (anharmonic) restoring forces, respectively. In principle these should also depend on $j$, but for simplicity in the following development we assume that the bonds within the unit cell are equivalent. In general $\kappa_{1}$ and $\kappa_{2}$ are tensors, but consistent with SBHM assumptions we treat them as scalars. The term $\kappa_{2}$ vanishes in the bulk for crystals with inversion symmetry, since the bonds there are symmetric. However, it is 
nonzero at surfaces and interfaces. We have ignored damping terms in Eq. (1f), since they can be added trivially.

We now perform the spatial and temporal integrals in Eq. (1a), obtaining

$$
\begin{aligned}
\vec{A}(\vec{r}, t)= & \frac{-i \omega q}{r c} e^{i k r-i \omega t} e^{-i \vec{k} \cdot \vec{r}_{o}} \\
& \times \sum_{j} e^{-i \vec{k} \cdot \Delta \vec{r}_{j}(t)} \hat{b}_{j}\left[\Delta r_{j 1}+2 \Delta r_{j 2} e^{i k r-i \omega t} e^{-i \vec{k} \cdot \vec{r}_{o}} e^{-i \vec{k} \cdot \Delta \vec{r}_{j}(t)}\right] .
\end{aligned}
$$

In Eq. (2) we substitute the complex wave vector $\vec{k}=\vec{k}(\omega)$ of the material for the free-space value, which is acceptable because we are considering optically isotropic materials at a discrete frequency $\omega$. The magnetic-dipole and bulkquadrupole terms ${ }^{10-12}$ arise from the expansion of the exponential next to the summation symbol, although in this application the magnetic-dipole contribution vanishes.

The next step is to evaluate Eq. (2) by iteration. We first determine $\Delta r_{j 1}$ by retaining only those terms proportional to $e^{-i \omega t}$. We evaluate $\Delta r_{j 2}$ in the same way, with the result for $\Delta r_{j 1}$ substituted where relevant. The connection between $\vec{A}(\vec{r}, t)$ and the radiation field $\vec{E}_{f f}$ in the far-field region follows as a special case of the Liénard-Wiechert potentials for an accelerated charge. We first evaluate the vector-potential part of

$$
\vec{E}_{f f}=-\frac{1}{c} \frac{\partial \vec{A}}{\partial t}-\nabla \phi
$$

then discard the longitudinal component (this is the effect of $-\nabla \phi)$. The result is

$$
\begin{aligned}
\vec{E}_{f f}= & \frac{q k^{2}}{r}(\widetilde{I}-\hat{k} \hat{k}) \cdot \sum \hat{b}_{j}\left\{\frac{q\left(\hat{b}_{j} \cdot \vec{E}_{o}\right)}{\kappa_{1}-m \omega^{2}} e^{i k r-i \omega t} e^{i\left(\overrightarrow{k_{o}}-\vec{k}\right) \cdot \vec{r}_{o}}\right. \\
& +\frac{q^{2}\left(\hat{b}_{j} \cdot \vec{E}_{o}\right)^{2}}{\left(\kappa_{1}-m \omega^{2}\right)^{2}\left(\kappa_{1}-4 m \omega^{2}\right)}\left[-i\left(\hat{b}_{j} \cdot \vec{k}\right)\left(\kappa_{1}-4 m \omega^{2}\right)\right. \\
& \left.\left.+2 i\left(\hat{b}_{j} \cdot \vec{k}_{o}\right)\left(\kappa_{1}-m \omega^{2}\right)-2 \kappa_{2}\right] e^{2 i k r-2 i \omega t} e^{2 i\left(\vec{k}_{o}-\vec{k}\right) \cdot \vec{r}_{o}}\right\},
\end{aligned}
$$

where the projection operator $(\tilde{I}-\hat{k} \hat{k})$ can also be written $(\hat{s} \hat{s}+\hat{p} \hat{p})$. The first term in braces is the linear-optic response, and the remaining three describe SHG. The physical origin of the three SHG terms are clear from the development: the first term, which involves the outgoing wave vector, is the electric-quadrupole contribution; the second term, which involves the incoming wave vector, arises from spatial dispersion; and the third term, which depends only on the anharmonic restoring force, is the contribution from asymmetric bonds. The intensity is proportional to the absolute square of the far field after all contributions have been taken into account.

We can cast Eq. (4) into a more useful form by writing

$$
\alpha_{1}=\frac{q}{\kappa_{1}-m \omega^{2}} \approx \frac{q}{\kappa_{1}},
$$

where, as shown below, $\alpha_{1}$ is related to the linear susceptibility. In what follows we ignore the $m \omega^{2}$ contribution to $\kappa_{1}-m \omega^{2}$ and $\kappa_{1}-4 m \omega^{2}$, which is reasonable since for our application the frequency of interest is relatively far from any resonance. Then Eq. (4) takes the form

$$
\begin{aligned}
\vec{E}_{f f}= & \frac{q k^{2}}{r}(\widetilde{I}-\hat{k} \hat{k}) \cdot \sum \hat{b}_{j}\left\{\alpha_{1}\left(\hat{b}_{j} \cdot \vec{E}_{o}\right) e^{i k r-i \omega t} e^{i\left(\vec{k}_{o}-\vec{k}\right) \cdot \vec{r}_{o}}\right. \\
& +\alpha_{1}^{2}\left(\hat{b}_{j} \cdot \vec{E}_{o}\right)^{2}\left[-i\left(\hat{b}_{j} \cdot \vec{k}\right)+2 i\left(\hat{b}_{j} \cdot \vec{k}_{o}\right)\right. \\
& \left.\left.-\frac{2 \kappa_{2}}{\kappa_{1}}\right] e^{2 i k r-2 i \omega t} e^{2 i\left(\vec{k}_{o}-\vec{k}\right) \cdot \vec{r}_{o}}\right\} .
\end{aligned}
$$

Thus the dipole-forbidden bulk terms can be written in terms of the linear polarizability, as previously noted by Mendoza and Mochán. ${ }^{11}$

To obtain both linear and SHG fields we must sum Eq. (6) over the bonds in the unit cell and over unit cells. We consider first the sum over layers normal to the surface. Since the interface is essentially only one monolayer thick, its contribution to the SHG field can be evaluated directly without needing to perform a layer sum. For the linear and SHG bulk contributions we approximate this sum by an integral, finding for the linear case

$$
\sum_{l} e^{i\left(\vec{k}_{o}-\vec{k}\right) \cdot \vec{r}_{o}} \cong \frac{1}{\Lambda} \int_{-\infty}^{0} d z e^{-i k_{z} z}=\frac{i}{2 \Lambda k_{o z}}
$$

where $\Lambda$ is the interlayer spacing. This expression follows by assuming that the wave vectors of the incident and radiating waves are normal to the surface and point in opposite directions. The first assumption is valid to a high degree of accuracy for semiconductors, where refractive indices $n$ are of the order of 3 to 4 . Given this, the second assumption is clearly correct for the backscattering configuration. The above result also supposes that the complex refractive indices at the pump and SHG wavelengths are equal, which is an acceptable approximation for $800 \mathrm{~nm}$ pump radiation and a crystalline $\mathrm{Si}$ target. The SHG result differs in that the exponent is $-i 2 k_{i} z$, leading to a replacement of the factor of 2 on the right-hand side by 4 .

Performing a lateral integration over the surface leads to total linear and SHG contributions of

$$
\begin{aligned}
\vec{E}_{f f}^{t o t}= & \frac{\pi q}{V}(\widetilde{I}-\hat{k} \hat{k}) \cdot \sum_{j} \hat{b}_{j}\left\{\alpha_{1}\left(\hat{b}_{j} \cdot \vec{E}_{o}\right) e^{i k z-i \omega t}+i k \alpha_{1}^{2}\left(\hat{b}_{j} \cdot \vec{E}_{o}\right)^{2}\right. \\
& \left.\times\left(\frac{3}{4}\left(\hat{k} \cdot \hat{b}_{j}\right)-2 \Lambda \frac{\kappa_{2}}{\kappa_{1}}\right) e^{i 2 k z-i 2 \omega t}\right\},
\end{aligned}
$$

where $V=\Lambda A$ is the volume of the unit cell. For tetrahedral bonding the vector projections and the sum over $j$ reduce to $4 / 3$ in the linear case, whence at normal incidence the linear term reduces to approximately 


$$
\frac{E_{f f}}{E_{o}} \approx \frac{4 \pi q \alpha_{1}}{3 V}=\frac{1-n}{1+n} .
$$

We can thus relate the SHG intensity originating from the bulk to the intensity $I_{o}$ of the driving laser as

$$
I_{S H G} \approx \frac{\pi \gamma^{2} V^{2} I_{o}^{2}}{128 \lambda^{2} c q^{2}}\left(\frac{1-n}{1+n}\right)^{4}
$$

where

$$
\gamma=\sum_{j}\left[(\widetilde{I}-\hat{k} \hat{k}) \cdot \hat{b}_{j}\right]\left(\hat{k} \cdot \hat{b}_{j}\right)\left(\hat{b}_{j} \cdot \hat{E}_{j}\right)^{2}
$$

is the vector summarizing SHG geometric factors and has a magnitude of the order of 0.2 . Using values appropriate to our SHG configuration (1 W average incident power, $\lambda=800 \mathrm{~nm}$, a duty cycle of $10^{-4}$, and an illuminated area of characteristic dimension $40 \mu \mathrm{m}$ ), Eq. (10b) leads to an estimate of $N_{S H G} \sim 10^{4} \gamma^{2} / \mathrm{s} \sim 0.04 \times 10^{4} / \mathrm{s}$, where $N_{S H G}$ is the number of bulk SHG photons generated per second. For comparison we typically observe total SHG generation rates of the order of $2.5 \times 10^{4} / \mathrm{s}$, which indicates that the bulk contribution, treated as if the intensities were additive, is very small.

An alternate estimate based directly on Eq. (4) leads to the same conclusion. Taking the literature value ${ }^{13} \chi_{1}$ $=1.06 \times 10^{-10}$ for $\mathrm{Si}$, assuming an incident field of the order of $1 \times 10^{8} \mathrm{~V} / \mathrm{m}$, and using $\lambda=800 \mathrm{~nm}$, a unit cell volume $=4 \times 10^{-23} \mathrm{~cm}^{3}$, a sample-collector separation of $0.5 \mathrm{~m}$, a $2 \omega$ absorption coefficient of $9.0 \times 10^{4} \mathrm{~cm}^{-1}$, and a volume exposed to the incident radiation of $1.4 \times 10^{-10} \mathrm{~cm}^{3}$, we find the SHG energy emitted to be about $0.24 \times 10^{-14} \mathrm{~J} / \mathrm{s}$. This can be compared to values of about $1 \times 10^{-14} \mathrm{~J} / \mathrm{s}$ that we measure experimentally. Hence from this perspective the bulk contributes less than about one-fourth of the observed SHG intensity.

SHG anisotropy data provide another source of information. We consider first the results of Lüpke et al., ${ }^{9}$ which include all four polarization combinations $p p, p s, s p$, and $s s$, and are shown in Fig. 1. These data were obtained at a wavelength of $765 \mathrm{~nm}$ on a Si sample cut $5^{\circ}$ off (111) toward the [11-2] direction. The angles of incidence and observation in air were both $45^{\circ}$. To obtain the relative bulk contribution we performed a least-squares analysis, fitting all four data sets simultaneously. The individual polarization combinations are normalized to the sixth harmonic of the Fourier decompositions, ${ }^{9}$ so their actual magnitudes are unknown. We take this into account by using individual scaling factors for the $p s, s p$, and $s s$ data, which would also contain the Fresnel factors if the intensities were calibrated. However, we use a common scaling factor between the interface and bulk contributions for all four data sets, since this is independent of measurement conditions. The remaining adjustable parameters are the hyperpolarizabilities of the up, step, and back bonds of the interface contribution, where the imaginary part of the hyperpolarizability of one of the two back bonds is assumed to be zero, and hence used as a phase reference. The fixed parameters are the vicinal angle of $5^{\circ}$, the angles of incidence of $29.1^{\circ}$ and $11^{\circ}$ for the interface and

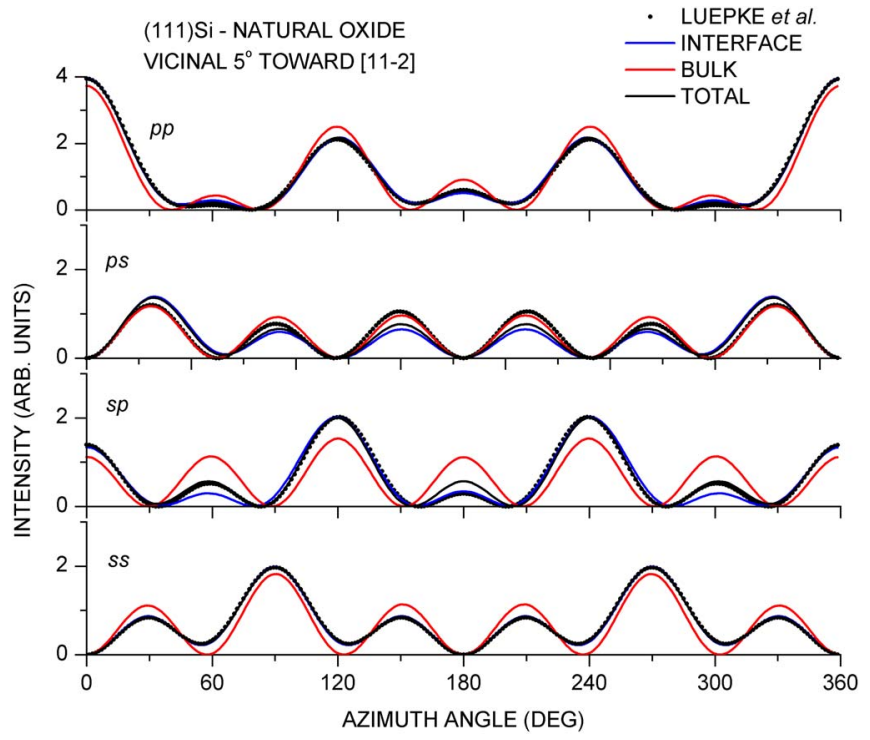

FIG. 1. (Color online) Azimuth dependence of SHG for vicinal Si $5^{\circ}$ off (111) toward [11-2].

bulk, respectively, and the bond angle of $109.4^{\circ}$ for both interface and bulk. In particular we use the specified vicinal angle of $5^{\circ}$ instead of the value of $1.12^{\circ}$ that we used previously. The angles of incidence are obtained from Snell's law, taking into account the refractive indices of $\mathrm{SiO}_{2}$ and $\mathrm{Si}$.

The results are shown in Fig. 1. The residuals obtained when all four data sets are fit simultaneously are 0.263 , 0.116 , and 0.087 for bulk-only, interface-only, and bulk and interface combined. In the last case the separate bulk and interface contributions for the $p p$ combination are shown in Fig. 2. The fact that the total intensity is not simply the sum of the individual intensities is due to coherency effects: when both bulk and interface contributions are present, the intensities are calculated only after adding the fields. The residuals indicate that the overall fit improves substantially through

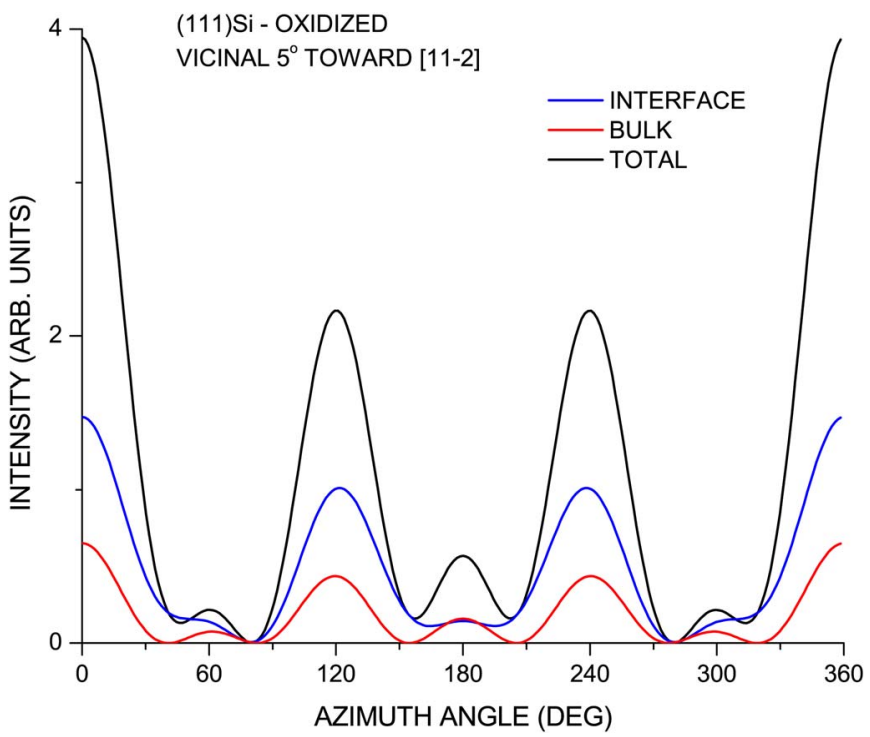

FIG. 2. (Color online) Different contributions to the $p p$ data of Fig. 1. 


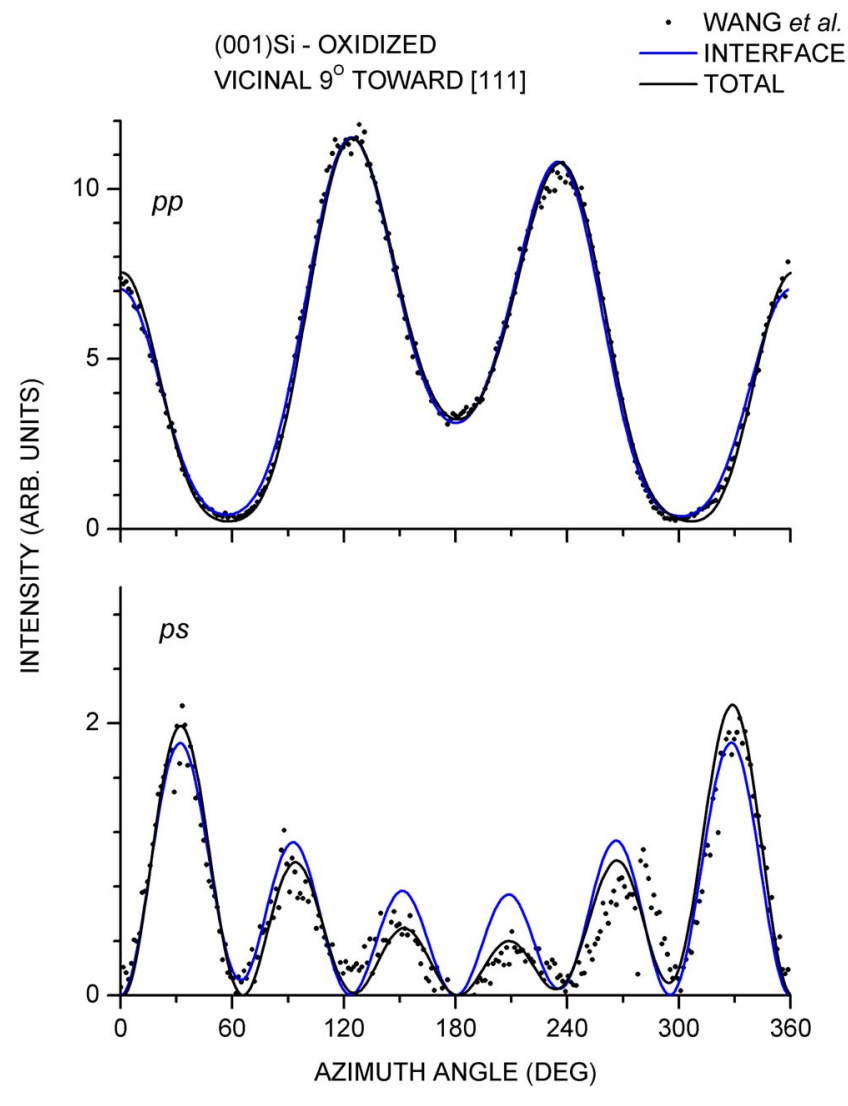

FIG. 3. (Color online) Azimuth dependence of SHG for vicinal Si $9^{\circ}$ off (001) toward [111].

the sequence. The surprising result is that the bulk contribution, for which the line shapes are invariant and the only parameters that can be varied are the scaling factors, provides such a good representation of the data. A better fit is obtained if we assume that SHG arises entirely from the interface. The interface hyperpolarizabilities obtained in this case are $1.28+i 0.28,3.11+i 1.66,2.23-i 0.01$, and $2.23+i 0$, where the last is used as a phase reference. The values are considered dimensionless because only relative amplitudes and phases are relevant. With both bulk and interface contributions included the fit improves further. As shown in Fig. 2, on an individual-intensity basis the bulk contributes about one-quarter of the overall signal, and the interface hyperpolarizabilities are found to be $1.19+i 0.81,1.45+i 1.39$, $1.42-i 0.00$, and $1.42-i 0$. Thus the bulk contribution causes the interface parameters to adjust to improve the overall fit, mostly via a change of the hyperpolarizability of the second, "step" bond. Since all combinations provide an acceptable representation of the observed anisotropies, the (111) data are somewhat inconclusive.

A more definitive example is given in Fig. 3. These data were obtained on a (001)Si surface cut $9^{\circ}$ off (001) toward [111]. ${ }^{6}$ As shown previously, ${ }^{7}$ the angle of incidence appropriate to this interface contribution is $12^{\circ}$. The bulk contributions, shown in Fig. 4, are very different from the data, in particular contributing virtually nothing to the major intensity peaks near 0 and $30^{\circ}$ in the $p p$ and $p s$ data, respectively. Hence, and in contrast to the vicinal (111) case, a bulk-only representation cannot succeed and was not attempted. Thus

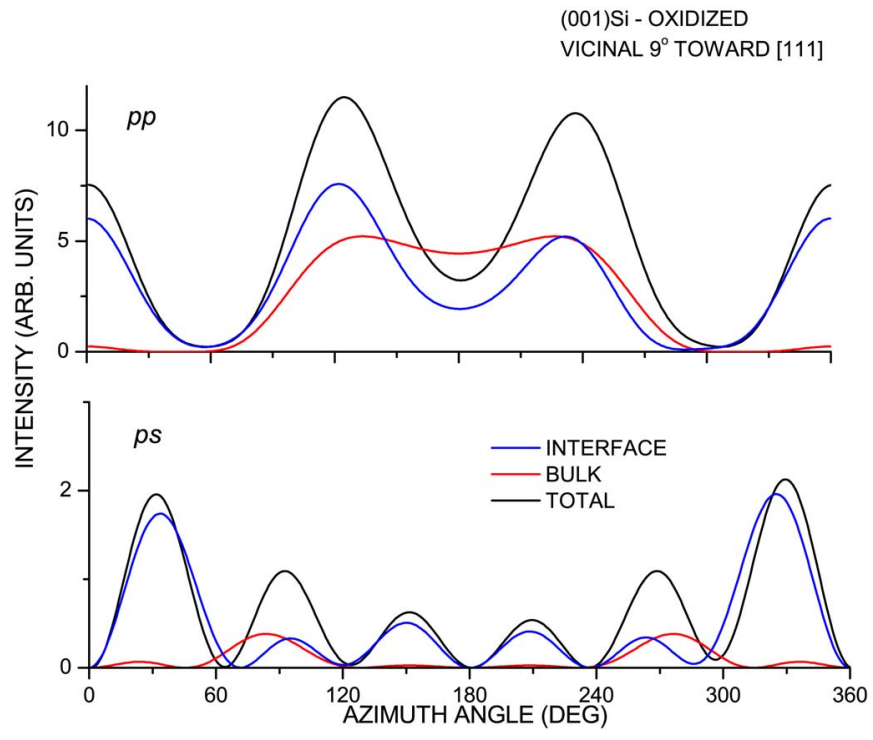

FIG. 4. (Color online) Different contributions to the data of Fig. 3.

no bulk traces are shown in Fig. 3. The residuals for interface-only and combined interface and bulk contributions are 29.9 and 18.8, respectively. For the interface-only case the interface hyperpolarizabilities are 87.9-i0.9, $188.5-i 47.9,122.8+i 0$, and $120.9+i 0$, with the last being used as the phase reference. With the bulk included the values become 67.2-i2.4, 149.5-i34.8, 90.0+i6.5, and 84.9 $+i 0$, so all hyperpolarizabilities change by similar amounts. From Fig. 3 the improvement observed with the bulk included arises almost entirely from a better representation of the $p s$ data, which in turn results from the coherent addition of a small bulk contribution to the interface contribution for the peaks at $30^{\circ}$ and $330^{\circ}$, and a coherent subtraction for the four interior peaks. The overall $p p$ simulation is essentially unaffected, even though Fig. 4 indicates that its bulk and interface contributions are now nominally equal. However, the good fit obtained in the interface-only case and the substantial differences between the bulk line shapes and data suggest that the improvement observed with the bulk contribution included may only be a secondary effect, with the bulk acting to artificially distort the interface contribution to give the better overall fit. In any case, the improved fit is a direct consequence of the coherent superposition of the interface and bulk signals, so the coherent superposition cannot be ignored.

One of the difficulties in analyzing the Lüpke et al. ${ }^{9}$ data follows from the absence of information about the $7 \phi$ Fourier coefficients. Our SBHM simulations show that the $7 \phi$ coefficients appear only if the bulk contribution is present and the surface orientation is neither exactly (001) nor (111). Thus both a bulk contribution and a vicinal orientation are necessary to generate a $7 \phi$ term. For small vicinal angles, which describe the cases considered here, we can use the magnitude of $7 \phi$ coefficients to estimate the bulk contribution. For the (001)Si $p p$ data these coefficients are less than $10 \%$ of the $6 \phi$ coefficients, providing further evidence that the bulk contribution is small. 
In conclusion, we have estimated the relative bulk and interface contributions to SHG for oxidized Si by obtaining an analytic expression for the first-forbidden bulk contributions in the SBHM, evaluating this expression directly using reasonable experimental parameters, then performing a leastsquares analysis of SHG anisotropy data for vicinal (111) and (001)Si samples. The fact that the SBHM describes observed SHG anisotropies for all polarization combinations is consistent with previous results for bulk THG and interface FHG data, and lends further credence to the model. Of course, a critical quantitative test of the model would require the force constants, and therefore the hyperpolarizabilities, to be calculated from first principles, which should not be be- yond the capabilities of a competent theorist. From the present results we conclude that the major fraction of observed nonlinear-optical anisotropies arises from bond geometry and therefore, as with linear optics, very little information is available from single-wavelength data. Nevertheless, even in the absence of spectral data the SBHM allows us to clarify previous assumptions about the relative importance of bulk and interface contributions to SHG in centrosymmetric materials, identify the importance of the interaction between bulk and surface contributions, and recognize the importance of the $7 \phi$ component in determining bulk contributions for vicinal samples. We expect these results to stimulate further work in this area.
*Corresponding author. Email: hpeng@ncsu.edu

${ }^{1}$ T. V. Murzina, R. V. Kapra, and O. A. Aktsipetrov, Appl. Phys. Lett. 83, 4749 (2003).

${ }^{2}$ M. Fiebig, D. Fröhlich, Th. Lottermoser, and M. Maat, Phys. Rev. B 66, 144102 (2002).

${ }^{3}$ V. Fomenko and E. Borguet, Phys. Rev. B 68, 081301(R) (2003).

${ }^{4}$ J. S. Salafsky and K. B. Eisenthal, J. Phys. Chem. B 104, 7752 (2000).

${ }^{5}$ G. D. Powell, J.-F. Wang, and D. E. Aspnes, Phys. Rev. B 65, 205320 (2002).

${ }^{6}$ J.-F. T. Wang, G. D. Powell, R. S. Johnson, G. Lucovsky, and D. E. Aspnes, J. Vac. Sci. Technol. B 20, 1699 (2002).
${ }^{7}$ H. J. Peng and D. E. Aspnes, Phys. Rev. B 70, 165312 (2004).

${ }^{8}$ J.-K. Hansen, H. J. Peng, and D. E. Aspnes, J. Vac. Sci. Technol. B 21, 1798 (2003).

${ }^{9}$ G. Lüpke, D. J. Bottomley, and H. M. van Driel, J. Opt. Soc. Am. B 11, 33 (1994).

${ }^{10}$ J. D. Jackson, Classical Electrodynamics, 3rd ed. (Wiley, New York, 1999).

${ }^{11}$ B. S. Mendoza and W. L. Mochán, Phys. Rev. B 55, 2489 (1997).

${ }^{12}$ N. Bloembergen, R. K. Chang, S. S. Jha, and C. H. Lee, Phys. Rev. 174, 813 (1968).

${ }^{13}$ D. E. Aspnes and A. A. Studna, Phys. Rev. B 27, 985 (1983). 